\title{
DOSES E FORMAS DE APLICAÇÃO DE SELÊNIO NA CULTURA DA SOJA ${ }^{1}$
}

\author{
Doses and forms of selenium application on the culture of soybean
}

\author{
Roberto Antonio Savelli Martinez ${ }^{2}$, Pedro Milanez de Rezende ${ }^{3}$, Amauri Alves de Alvarenga ${ }^{4}$, \\ Messias José Bastos de Andrade ${ }^{5}$, Alexandre Martins Abdão dos Passos ${ }^{6}$
}

\begin{abstract}
RESUMO
Objetivou-se, nesse trabalho, determinar os efeitos de doses e formas de aplicação de selênio na forma de selenito de sódio na produtividade e características agronômicas da soja [Glycine max (L) Merrill]. Utilizou-se um delineamento experimental em blocos ao acaso, com um esquema fatorial de $4 \times 3+(1)$ compreendendo 4 doses de selênio $\left(0,5 ; 1,0 ; 1,5 ; 2,0 \mathrm{~kg} \mathrm{ha}^{-1}\right)$, três formas de aplicação (solo, foliar e solo + foliar) mais uma testemunha sem adubação, com três repetições. No tratamento solo + foliar aplicou-se a metade de cada dose isolada. A aplicação do selênio no solo foi feita na semeadura em mistura com os macronutrientes, e a aplicação foliar foi realizada no estágio V8 da cultura. As parcelas foram constituídas de 4 linhas com 5 metros de comprimento, espaçadas em 50 centímetros, com uma densidade de 12 plantas por metro linear. A adubação com selenito de sódio via foliar, independentemente das doses, afeta altura da planta, altura da inserção do primeiro legume e número de sementes por legume, promovendo um efeito fitotóxico na planta, chegando a reduzir em até $21 \%$ a produtividade. O número médio de legumes por planta e a massa de 100 sementes não sofreram efeitos da adubação com selênio.
\end{abstract}

Termos para indexação: Glycine max, selênito de sódio, fitotoxidez, produtividade de grãos.

\begin{abstract}
The aim of this work was to determine the effects of doses and forms of selenium applications in the form of sodium selenide on the productivity and agronomic characteristics of the soybean [Glycine max (L) Merrill]. An experimental design in randomized blocks was used with a factorial outline of $4 \times 3(+1)$, four selenium doses $\left(0.5,1.0,1.5\right.$, and $\left.2.0 \mathrm{~kg} . \mathrm{ha}^{-1}\right)$, three application forms (soil, foliar and soil + foliar), and a control, with three replications. In the treatment soil + foliar, half of each dose isolated was applied. The application of selenium to the soil was made in the planting process and the application to the leaves was accomplished in the V8 stage of the culture. The parcels were composed of four lines with five meters in length, spaced 50 centimeters from each other, with a density of 12 plants for each linear meter. The fertilization, with sodium selenide via leaves, independently of the doses, affected the characteristics height of the first pod insertion, height of the plant, and number of seeds in the pods, due to a phytotoxic effect, decreasing the productivity in $21 \%$. The average number of pods per plant and the average weight of 100 seeds were not affected by the fertilization with selenium.
\end{abstract}

Index terms: Glycine max, sodium selenide, phytotoxicity, yield

(Recebido em 2 de agosto de 2007 e aprovado em 4 de abril de 2008)

\section{INTRODUÇÃO}

A nutrição mineral constitui um dos principais fatores da produção agrícola, sobretudo quando se considera os níveis de macro e de micronutrientes adicionados ao solo no plantio ou em pulverização foliar (LAVIOLA et al., 2007; EPSTEIN, 1975). Particularmente no caso do selênio, os teores desse elemento nas plantas, animais e seres humanos, numa determinada região, estão diretamente relacionados com o conteúdo desse elemento presente no solo, que por sua vez está relacionado com a pedologia, gênese e localização nas áreas de cultivo e pastagem (ANDERSON et al., 1961). Nas plantas, o selênio apresenta propriedades químicas semelhantes às do enxofre, estando presente em aminoácidos sulfurados como selenometionina e selenocisteína. As plantas apresentam capacidades diferenciadas de absorção e acumulação de Se do solo, sendo classificados como

${ }^{1}$ Parte da dissertação apresentada à Universidade Federal de Lavras / UFLA- Caixa postal 37200-000- Lavras, MG pelo primeiro autor,para obtenção do grau de mestre em agronomia, área de concentração fitotecnia. 2007

Engenheiro Agrônomo, Mestre - Departamento de Agricultura/DAG - Universidade Federal de Lavras/UFLA - Cx. P. 3037 - $37200-000$ - Lavras, MG rasmartinez@gmail.com

${ }^{3}$ Engenheiro Agrônomo, Doutor, Professor - Departamento de Agricultura/DAG - Universidade Federal de Lavras/UFLA - Cx. P. 3037 - $37200-000$ Lavras, MG - pmrezend@ufla.br

${ }^{4}$ Engenheiro Agrônomo, Doutor, Professor - Departamento de Biologia/DBI - Universidade Federal de Lavras/UFLA - Cx. P. 3037 - $37200-000$ - Lavras, MG amauriaa@ufla.br

${ }^{5}$ Engenheiro Agrônomo, PhD, Professor - Departamento de Agricultura/DAG - Universidade Federal de Lavras/UFLA - Cx. P. 3037 - $37200-000$ Lavras, MG - mandrade@ufla.br

${ }^{6}$ Mestre - Departamento de Agricultura/DAG - Universidade Federal de Lavras/UFLA - Cx. P. 3037 - 37200-000 - Lavras, MG - aabdao@terra.com.br 
acumuladoras e não acumuladoras de Se. Em plantas não acumuladoras, como é o caso da soja, o limite de concentração desse elemento é o de no máximo $6 \mu \mathrm{g}^{-1}$ ainda que cultivado em solos seleníferos (WHANGER, 2002).

De acordo com Turakainem et al. (2005), o selênio tem a propriedade de promover um reforço na capacidade das plantas de combater o estresse oxidativo causado por radicais livres do oxigênio. Por outro lado, a presença de altas concentrações na planta causa toxidez e ativa reações oxidativas. Nessa situação, as plantas se defendem do excesso de selênio produzindo compostos voláteis. No solo, esse elemento é encontrado principalmente como selenato e selenito, embora possam existir, também, selênio elementar, seleneto e formas orgânicas de selênio (KABATA-PENDIAS \& PENDIAS, 1999).

Estudos indicam a necessidade da suplementação dos fertilizantes com selênio desde 1984. Em 1998, o Ministério de Agricultura e Florestas Finlandês aumentou o nível de suplementação de selênio nos fertilizantes devido à baixa concentração desse elemento no solo, nos fertilizantes e nos alimentos (EUROLA et al., 2003).

No Brasil, trabalhos com esse elemento são escassos, existindo ainda um indicativo de baixo consumo, portanto é importante manter-se atento à possibilidade de deficiências de selênio na população brasileira, particularmente nos grupos de baixo poder aquisitivo que não consomem produtos de origem animal com freqüência (FERREIRA et al., 2002). São necessários estudos mais específicos para sua introdução nos alimentos, na tentativa de serem definidas, algumas estratégias como doses, formas de aplicação, bem como o conteúdo desse elemento nos alimentos, animais e humanos, proporcionando um consumo adequado desse nutriente .

A soja [Glycine max (L) Merrill], uma das culturas mais importantes na atualidade constitui uma excelente fonte de proteína vegetal sendo parte integrante de grande grupo dos alimentos consumidos na alimentação humana e animal, foi tomada como referência no desenvolvimento desse trabalho.

Objetivou-se, no trabalho de pesquisa, estudar o efeito de doses e formas de aplicação de selênio nas características agronômicas da soja e na produtividade de grãos.

\section{MATERIALE MÉTODOS}

O experimento foi conduzido na Fazenda Milanez localizada no município de Itutinga-MG, que se encontra à latitude de $21^{\circ} 23^{\prime} 29,8^{\prime \prime}$ Sul, longitude de 04439'13,2" Oeste e altitude média de $958 \mathrm{~m}$, no Estado de Minas Gerais - Brasil. O clima da região, baseado na classificação internacional de Köeppen, é do tipo Cwa, temperado úmido, com verão quente e inverno seco, caracterizado por uma pluviosidade de $23,4 \mathrm{~mm}$ no mês mais seco e de $295,8 \mathrm{~mm}$ no mês mais chuvoso. A temperatura média é de $22,1^{\circ} \mathrm{C}$ no mês mais quente e de $15,8^{\circ} \mathrm{C}$ no mês mais frio, sendo a precipitação média anual de 1529,7 mm (BRASIL, 1992).

O solo utilizado foi do tipo Cambissol, com as seguintes características químicas (Tabela 1).

A adubação de semeadura foi feita de acordo com a análise de solo e as interpretações de acordo com Ribeiro et al. (1999), sendo utilizado $400 \mathrm{~kg} \mathrm{ha}^{-1} \mathrm{da}$ formula 4-30-10.

As sementes de soja, cultivar MG/ BR6 (Conquista), foram inoculadas antes da semeadura com Bradyrhizobium japonicum, utilizando-se inoculante na proporção de 1.200.000 bactérias por semente.

O delineamento experimental utilizado foi o de blocos ao acaso com três repetições, esquema fatorial de $4 \times 3+1$, constituído de 4 doses de selênio $(0,5 ; 1,0 ; 1,5 ; 2,0$ $\mathrm{kg} \mathrm{ha}^{-1}$ ), três formas de aplicação (solo, foliar e solo + foliar), mais uma testemunha sem adubação; no tratamento solo + foliar a dose foi dividida, sendo metade aplicada no solo por ocasião do plantio e a outra metade via foliar realizada

Tabela 1 - Características químicas do solo da área experimental.

\begin{tabular}{|c|c|c|c|c|c|c|c|c|c|c|}
\hline \multirow[t]{2}{*}{$\begin{array}{c}\mathrm{pH} \\
\mathrm{H}_{2} \mathrm{O}\end{array}$} & & & \multicolumn{7}{|c|}{$\mathrm{Cmol}_{\mathrm{c}} \mathrm{dm}^{-3}$} & \multirow{2}{*}{$\begin{array}{l}\% \\
\mathrm{~V}\end{array}$} \\
\hline & $\mathrm{P}$ & $\mathrm{K}$ & $\mathrm{Ca}$ & $\mathrm{Mg}$ & $\mathrm{Al}$ & $\mathrm{H}+\mathrm{Al}$ & SB & $\mathrm{T}$ & $\mathrm{T}$ & \\
\hline 6,1 & 2,5 & 129 & 3,0 & 1,0 & 0,0 & 1,7 & 4,3 & 4,3 & 6,0 & 71,8 \\
\hline $\operatorname{dag} \mathrm{Kg}^{-1}$ & & $\mathrm{mg} \mathrm{L}^{-1}$ & \multicolumn{8}{|c|}{$\mathrm{mg} \mathrm{dm}{ }^{-3}$} \\
\hline MO & & Prem & $\mathrm{Zn}$ & $\mathrm{Fe}$ & & Mn & $\mathrm{Cu}$ & & B & S \\
\hline 3,6 & & 9,9 & 0,8 & 46,6 & & 5,1 & 1,4 & & 0,4 & 22,7 \\
\hline
\end{tabular}


no estádio V8 da cultura, que ocorreu 45 dias após a emergência das plantas. As aplicações no solo foram realizadas misturando-se selênio utilizado na forma de selenito de sódio ao adubo de semeadura relatado acima. A aplicação foliar foi realizada com auxílio de um pulverizador a gás carbônico de pressão constante de 2,8 kgf. $\mathrm{cm}^{-2}$, utilizando um volume de calda de 2001.ha ${ }^{-1}$.

A semeadura foi realizada no dia 23 de novembro de 2005 , a uma profundidade de $2 \mathrm{a} 3 \mathrm{~cm}$, após ter sido feito o preparo de solo de forma convencional.

As parcelas foram compostas de 4 linhas com 5,0 metros de comprimento, espaçadas de 0,5 metro, sendo as duas fileiras externas as bordaduras e as centrais as fileiras úteis. Todos os tratamentos receberam, sempre que necessário, os tratos culturais como capinas realizadas de forma manual, controle de lagartas com a utilização de Decis $0,151 \cdot \mathrm{ha}^{-1} \mathrm{e}$ ferrugem utilizando Ópera $0,51 \cdot \mathrm{ha}^{-1}$. A fonte de selênio utilizada foi selenito de sódio $\left(\mathrm{Na}_{2} \mathrm{SeO}_{3}\right)$ com $450 \mathrm{~g}$ de selênio por quilo do produto, aplicado tanto no solo quanto na adubação foliar da cultura. As seguintes características foram analisadas: produtividade de grãos, massa de 100 sementes, número de legumes por planta, número de sementes por legume, altura do primeiro legume, altura da planta e acamamento. A produtividade foi determinada colhendo-se as plantas das duas fileiras centrais úteis, que após beneficiadas em trilhadora de parcelas experimentais, tiveram a umidade dos grãos padronizados para $13 \%$ e transformadas em $\mathrm{kg} \mathrm{ha}^{-1}$. A altura do primeiro legume, altura da planta, número de legumes por planta, número de grãos por legume e massa de 100 sementes, foram determinados em cada parcela tomandose aleatoriamente 10 plantas das fileiras úteis. $\mathrm{O}$ grau de acamamento foi determinado em cada parcela, atribuindose notas de 1 até 5 , de acordo com a escala proposta por Bernard et al. (1965) sendo 1 todas as plantas eretas, e 5 todas as plantas acamadas. A emergência ocorreu no dia 29/11/2005 de uma maneira uniforme, o mesmo se verificando em relação à floração em 11/01/2006; aos 11 dias após emergência foi realizado o desbaste deixando 12 plantas por metro linear de acordo com Rezende et al. (1982).

Os dados foram analisados com o programa "Statistical Analysis System SASâ", sendo os mesmos submetidos ao teste F para análise de variância e os efeitos dos tratamentos, quando significativos, foram submetidos ao teste de Tukey, a 5\% e 1\% e, nos casos de efeitos de doses, foram submetidos à analise de regressão.

\section{RESULTADOS E DISCUSSÃO}

A altura das plantas, altura de inserção do primeiro legume e número de sementes por legume apresentaram efeitos significativos para a variável formas de aplicação. Produtividade de grãos apresentou efeito significativo, quando se compara a média dos componentes do fatorial com o tratamento adicional. O número de legumes por planta e o peso de cem sementes não mostraram efeitos dos tratamentos (Tabela 2).

Tabela 2 - Análise de variância para altura de plantas (ALTPLAN), altura de inserção do primeiro legume (ALTLEG), número de legumes por planta (NLPLAN), número de sementes por legume (NSEMLEG), produtividade de grãos (PROD), massa de 100 sementes (MCS), obtidas no experimento doses e formas de aplicação de selênio na cultura da soja. Itutinga - MG 2005/2006.

\begin{tabular}{cccccccc}
\hline Causas variação & \multirow{2}{*}{ GL } & \multicolumn{7}{c}{ Quadrados médios } \\
\cline { 3 - 9 } & & ALTPLAN & ALTLEG & MCS & NLPLA & NSEMLEG & PROD \\
\hline Blocos & 2 & 28,99 & 9,13 & 22,24 & 165,60 & 0,65 & 961761,54 \\
Formas & 2 & $370,66^{*}$ & $54,01^{*}$ & 0,93 & 4,35 & $0,75^{*}$ & 272133,33 \\
(F) & 3 & 102,67 & 8,18 & 0,11 & 80,04 & 0,08 & 189222,22 \\
Doses (D) & 6 & 116,24 & 8,09 & 0,6 & 70,52 & 0,084 & 192922,22 \\
F x D & 1 & 273,37 & 0,03 & 2,31 & 19,37 & 0,14 & $673969,18^{*}$ \\
Fatorial & & & & & & & \\
$\quad$ x & 24 & 78,75 & 14,14 & 0,66 & 64,61 & 0,17 & 125250,43 \\
\hline adicional & & $13,27 \%$ & $19,23 \%$ & $4,99 \%$ & $24,10 \%$ & $16,73 \%$ & $19,08 \%$ \\
\hline Resíduo & & & & & & &
\end{tabular}

* significativos a 5\% pelo teste de F. 
A altura da planta e altura de inserção do primeiro legume foram alteradas pelas formas de aplicação de selênio, apresentando variações entre 50 e $74 \mathrm{~cm}$ para altura da planta e de 16 a $23 \mathrm{~cm}$ para altura do primeiro legume (Tabela 3). Esses valores podem ser considerados dentro dos padrões para colheita mecânica, uma vez que as colhedoras atuais conseguem realizar essa operação em cultivares de soja cuja inserção do primeiro legume esteja acima de $10 \mathrm{~cm}$ (Tabela 3 ).

Não foram verificadas diferenças significativas na altura das plantas quando a aplicação de selênio foi feita via solo ou na combinação via foliar mais solo (Tabela 3). Já no tratamento em que utilizou-se apenas a adubação via foliar com selênio, o desenvolvimento das plantas foi afetado, o que pode ser explicado por uma fitotoxidez ocasionada nas plantas por ocasião da aplicação do produto. É importante ressaltar que, independente da dose, as aplicações foliares de selênio proporcionaram o aparecimento do efeito fitotóxico(queima das folhas) nas plantas, sendo esse crescente com o aumento das doses. Esses resultados podem ser devidos à aplicação da dose utilizada ou à concentração do elemento na calda, uma vez que o volume utilizado foi de apenas $2001 \mathrm{ha}^{-1}$. Convém salientar que todas as plantas submetidas ao tratamento foliar recuperaram-se visualmente, com o transcorrer do ciclo. Em relação à aplicação no solo não se constatou, aparentemente, nenhum efeito fitotóxico na cultura.

No caso da altura da inserção do primeiro legume, verificou-se também que a aplicação via foliar reduziu significativamente a inserção do primeiro legume, porém sem diferir da aplicação via solo. Os tratamentos solo e solo + foliar apresentaram comportamento semelhante não apresentando diferença significativa entre si (Tabela.3).

Normalmente, existe correlação positiva entre altura da planta e da inserção do primeiro legume, ou seja, plantas mais altas têm altura de inserção também mais elevada, além disso quando as plantas são expostas a altas concentrações de Se podem surgir sintomas de agressão como: atrofiamento do crescimento, clorose, murchamento , secamento das folhas, decréscimo da síntese de proteína e morte prematura da planta. Segundo Turakainen et al. (2005) a utilização de selênio reforça a capacidade das plantas de combater o estresse oxidativo causado por radicais livres do oxigênio. Entretanto, altas concentrações de selênio nas plantas causam toxidez e ativam reações oxidativas, além de aumentar a peroxidação lipídica . As plantas se defendem do excesso de selênio produzindo compostos voláteis desse elemento. Outro ponto a considerar é que existem diferenças entre as plantas acumuladoras e as não acumuladoras de Se. Em plantas não acumuladoras, o limite de concentração de Se máximo encontrado é de aproximadamente $6 \mu . \mathrm{g}^{-1}$ mesmo quando cultivado em solos selêniferos e aqui incluímos a soja (WHANGER, 2002); e em tecido meristemático resultou em uma redução de $10 \%$ em rendimento que variou de $2 \mathrm{mg}$ de $\mathrm{Se} \mathrm{kg}^{-1} \mathrm{em}$ arroz, para $330 \mathrm{mg}$ de $\mathrm{Se} \mathrm{kg}^{-1}$, em trevo branco (MIKKELSEN et al., 1988).

O número de legumes por planta apresentou variação de 27 a 40 e o número de sementes por legume de 2,0 a 2,87 (Tabela 4).

O número de legumes por planta não foi influenciado significativamente pelos tratamentos testados, o mesmo não sendo verificado para número de sementes por legume, que foi alterado significativamente pelas formas de aplicação do selênio. A aplicação via foliar apresentou pior desempenho em relação às outras duas formas de aplicação que não apresentaram diferenças significativas entre si, solo e solo + foliar (Tabela 4). Essa variação do número de sementes por legume talvez possa ter acontecido devido a um efeito residual da fitotoxidez que diminuiu a área fotossintética na planta por ocasião da aplicação do selênio.

A massa de 100 sementes apresentou uma variação de 15,54 a $16,85 \mathrm{~g}$ e a produtividade de grãos de 1220 a 2310 kg.ha-1 (Tabela 5).

No caso da massa de 100 sementes e da produtividade, as doses e formas de aplicação não alteraram significativamente essa característica. Efeitos significativos foram observados na produtividade para a interação fatorial versus adicional. Comparando-se a média dos tratamentos com a aplicação de selênio com a média dos adicionais, que não receberam aplicações de selênio, verifica-se aumento na produtividade em favor do adicional de $493 \mathrm{~kg} \mathrm{ha}^{-1}(27,13 \%)$. Esses resultados demonstram que a aplicação de selênio diminuiu a produtividade da soja, uma vez que a média da testemunha foi sempre superior à média dos tratamentos com selênio.

Levando-se em conta que ocorreu efeito fitotóxico da aplicação do selênio via foliar, ocorrendo queima das folhas e consequientemente diminuição da área foliar na cultura da soja, que é uma planta $\mathrm{C}_{3}$, esse fatopode provocar perdas na taxa assimilatória líquida, a qual representa o incremento em matéria seca por cada unidade de superfície de área foliar disponível à planta, durante um certo intervalo de tempo pré-determinado (HUNT et al., 2002). Com diminuição dessa área foliar ocorreu menor formação de fotoassimilados necessários ao enchimento de grãos, reduzindo assim a produtividade, e a redução no número de sementes por legume influenciou esse resultado já que ele é um dos componentes da produção (Tabela 4). 
Tabela 3 - Resultados em centímetros da altura das plantas (ALTPLAN) e da inserção do $1^{\circ}$ legume (ALTLEG) de soja, cv. Conquista obtidas no experimento doses e formas de aplicação de selênio. Itutinga - MG, 2005/2006.

\begin{tabular}{|c|c|c|c|c|c|c|c|c|}
\hline \multirow[t]{2}{*}{ Doses } & \multicolumn{2}{|c|}{$\begin{array}{c}\text { ALTPLAN } \\
\text { Formas }\end{array}$} & \multirow[b]{2}{*}{$\mathrm{S}+\mathrm{F}$} & \multirow[b]{2}{*}{ Média } & \multirow[b]{2}{*}{ Foliar } & \multicolumn{3}{|c|}{$\begin{array}{l}\text { ALTLEG } \\
\text { Formas }\end{array}$} \\
\hline & Foliar & Solo & & & & Solo & $\mathrm{S}+\mathrm{F}$ & Média \\
\hline 0,5 & 69 & 70 & 67 & 69 & 18 & 19 & 21 & 19 \\
\hline 1,0 & 64 & 74 & 70 & 69 & 18 & 21 & 23 & 21 \\
\hline 1,5 & 55 & 61 & 73 & 63 & 17 & 17 & 21 & 18 \\
\hline 2,0 & 50 & 73 & 67 & 63 & 16 & 23 & 22 & 20 \\
\hline Medias & 60B & $70 \mathrm{~A}$ & $69 \mathrm{~A}$ & 66 & 17B & $20 \mathrm{AB}$ & $22 \mathrm{~A}$ & 20 \\
\hline Tes & \multicolumn{4}{|c|}{76} & \multicolumn{4}{|c|}{19} \\
\hline
\end{tabular}

Médias seguidas da mesma letra não diferem em nível de 5\%, pelo teste de Tukey.

Tabela 4 - Número de legumes por planta (NLPLAN) e de sementes por legume (NSEMLEG), obtidos no experimento doses e formas de aplicação de selênio. Itutinga - MG, 2005/2006.

\begin{tabular}{|c|c|c|c|c|c|c|c|c|}
\hline \multirow[t]{2}{*}{ Doses } & \multicolumn{3}{|c|}{$\begin{array}{c}\text { NLPLAN } \\
\text { Formas }\end{array}$} & \multirow[b]{2}{*}{ Médias } & \multicolumn{4}{|c|}{$\begin{array}{c}\text { NSEMLEG } \\
\text { Formas }\end{array}$} \\
\hline & FOL & Solo & $\mathrm{S}+\mathrm{F}$ & & FOL & Solo & $\mathrm{S}+\mathrm{F}$ & Médias \\
\hline 0,5 & 40 & 37 & 35 & 37 & 2,13 & 2,46 & 2,33 & 2,31 \\
\hline 1,0 & 39 & 33 & 27 & 33 & 2,33 & 2,53 & 2,67 & 2,51 \\
\hline 1,5 & 27 & 31 & 35 & 31 & 2,13 & 2,47 & 2,67 & 2,42 \\
\hline 2,0 & 29 & 29 & 35 & 31 & 2,00 & 2,87 & 2,67 & 2,51 \\
\hline Medias & 34 & 33 & 35 & 37 & $2,15 \mathrm{~B}$ & $2,58 \mathrm{~A}$ & $2,59 \mathrm{~A}$ & $2,44 \mathrm{~B}$ \\
\hline Testemunh & & 36 & & & & & & \\
\hline
\end{tabular}

Médias seguidas da mesma letra não diferem em nível de 5\%, pelo teste de Tukey.

Tabela 5 - Massa de 100 sementes (MCS) em gramas, e produtividade de grãos $\left(\mathrm{kg} \cdot \mathrm{ha}^{-1}\right)$ obtidas no experimento doses e formas de aplicação de selênio. Itutinga - MG, 2005/2006.

\begin{tabular}{|c|c|c|c|c|c|c|c|c|}
\hline \multirow[b]{2}{*}{ Doses } & \multirow[b]{2}{*}{ Foliar } & \multicolumn{2}{|c|}{$\begin{array}{c}\text { MCS } \\
\text { Formas }\end{array}$} & \multirow[b]{2}{*}{ Médias } & \multicolumn{2}{|c|}{$\begin{array}{c}\text { PROD } \\
\text { Formas }\end{array}$} & \multirow[b]{2}{*}{$\mathrm{S}+\mathrm{F}$} & \multirow[b]{2}{*}{ Médias } \\
\hline & & Solo & $\mathrm{S}+\mathrm{F}$ & & Foliar & Solo & & \\
\hline 0,5 & 16,67 & 15,85 & 16,26 & 16,26 & 2023 & 1970 & 1847 & 1947 \\
\hline 1,0 & 15,57 & 16,14 & 16,26 & 15,99 & 1763 & 2237 & 1803 & 1934 \\
\hline 1,5 & 15,69 & 16,45 & 16,37 & 16,17 & 1580 & 1657 & 1757 & 1665 \\
\hline 2,0 & 15,54 & 16,23 & 16,85 & 16,21 & 1220 & 1870 & 2073 & 1721 \\
\hline Medias & 15,87 & 16,17 & 16,44 & 16,16 & 1647 & 1934 & 1870 & $1817 \mathrm{~B}$ \\
\hline Testemunha & & 18,07 & & & & $2310 \mathrm{H}$ & & \\
\hline
\end{tabular}

Médias seguidas da mesma letra não diferem entre si em nível de 5\%, pelo teste de Tukey.

Para o acamamento, todas as notas foram iguais ("1") verificando-se assim a ausência de variabilidade nessa característica, sendo essa nota caracterizada como todas plantas eretas.
Estudos realizados por Euliss \& Carmichael (2004), em canola (Brassica napus L), mostraram que as plantas têm crescimento hidropônico reduzido sob concentração de 2 ppm de selênio, 
repercutindo em queda no florescimento e no número de sementes.

A distribuição de Se nas várias partes da planta difere de acordo com as espécies, fase de desenvolvimento, e condições fisiológicas. Em acumuladoras de Se, esse é acumulado em folhas jovens, durante a fase vegetativa. Durante a fase reprodutiva, são encontrados níveis altos de Se em sementes, enquanto o seu conteúdo em folhas é drasticamente reduzido (WHO, 1982). Cereais não acumuladores, quando amadurecem, freqüentemente mostram o mesmo conteúdo de Se em grãos e raízes, com quantidades menores nos caules e folhas . A distribuição de Se em plantas também depende da forma e concentração e disponibilidade às raízes, natureza e concentração de outras substâncias, especialmente sulfatos acompanhando o elemento (SOUZA et al., 1998; ZAYED et al., 1998).

Em relação à massa de 100 sementes não foi verificada variação significativa para nenhum dos tratamentos realizados, não tendo assim nenhuma influência dos tratamentos, no acúmulo de matéria seca nas sementes (Tabela 5).

\section{CONCLUSÕES}

A aplicação exclusivamente via foliar de selênio, para todas as doses testadas, influenciou negativamente a altura da planta, altura do primeiro legume, número de sementes por legume e a produtividade da cultura da soja, cultivar Conquista.

\section{REFERÊNCIAS BIBLIOGRÁFICAS}

ANDERSON, M. S.; LAKIN, H. W.; BEESON, K. C.; FLOYD, F. S.; THUCKER, E. Selenium in agriculture. Washington, DC: Department of Agriculture, 1961. 65 p. (Agriculture handbook, 200).

BERNARD, R. L.; CHAMBERLAIN, D. W.; LAWRECE, R. D. (Eds.). Results of the cooperative uniform soybean tests. Washington, DC: USDA, 1965. $134 \mathrm{p}$.

BRASIL. Ministério da Agricultura e Reforma Agrária. Normais climatológicas: 1961-1990. Brasília, DF, 1992. 84 p.

EPSTEIN, E. Nutrição mineral de plantas: princípios e perspectivas; tradução e notas de E. Malavolta. Rio de Janeiro, Livros Técnicos e Científicos; São Paulo, Ed. da Universidade de São Paulo, 1975, 341 p.

EULISS, K. W.; CARMICHAEL, J. S. The effects of selenium accumulation in hydroponically grown canola (Brassica napus). Biological \& Biomedical Sciences,
North Dakota, v. 1, n. 10, Jan. 2004. Disponível em: $\Delta$ http:///www.jyi.org/volumes/volume10/issuel/articles/ euliss.html . Acesso em: 10 out. 2008.

EUROLA, M.; ALFTHAN, G.; ARO, A.; EKHOLM, P.; HIETAMNIEMI, V.; RAINIO, H.; RANKANEN, R.; VENALAINEN, E. Results of the Finnish selenium program 2000-2001. 2003. $45 \mathrm{f}$.

(Agrifoodresearch reports, 36). Disponível.em:

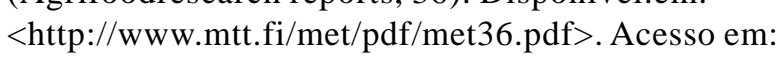
1 jul. 2005 .

FERREIRA, K. S.; GOMES, J. C.; BELLATO, C. R.; JORDÃO, C. P. Concentrações de selênio em alimentos consumidos no Brasil. Revista Panamericana de Salud Pública, Washington, v. 11, n. 3, 2002. Disponível em: 4http://www.scielosp.org/scielophp?pid.pt>1. Acesso em: 10 jun. 2005 .

HUNT, R.; CAUSTON, D. R.; SHIPLEY, B.; ASKEW, A. P. A modern tool for classical plant growth analisys: techical note. Annals of Botany, London, v. 90, p. 485288, 2002.

KABATA-PENDIAS, A.; PENDIAS, H. Biogeochemistry of trace elements in Polish. Warszawa: PWN, 1999.

LAVIOLA, B. G.; MARTINEZ, H. E. P.; MAURI, A. L. Influência do nível de adubação de plantas matrizes na formação de mudas de cafeeiros em sistema hidropônico. Ciência e Agrotecnologia, Lavras, v. 31, n.4, p.1043-1047, jul./ago., 2007.

MIKKELSEN, R. L.; PAGE, A. L.; HAGHNIA, G. H. Effect of salinity and its composition on the accumulation of selenium by alfalfa. Plant Soil, v. 107, p. 63-67, 1988.

REZENDE, P. M. de; BUENO, L. C. S.; SEDIYAMA, T.; JUNQUEIRA NETO, A.; LIMA, L. A. de P.; FRAGA, A. C. Épocas de desbaste em experimento com soja [Glycine max (L.) Merrill] em diferentes densidades de semeadura. In: SEMINÁRIO NACIONAL DE PESQUISA DE SOJA, 2., 1981, Brasília, DF. Anais... Londrina: Embrapa-CNPSo, 1982. v. 1, p. 201-206.

RIBEIRO, A. C.; GUIMARÃES, P. T. G.; ALVAREZ, V. H. Recomendações para o uso de corretivos e fertilizantes em Minas Gerais: $5^{\text {a }}$ aproximação. Viçosa: UFV, 1999. 359 p. 
SOUZA, M. P.; PILON-SMITS, E. A. H.; LYTLE, C. M. L.; HWANG, S.; TAI, J.; HONMA, T. S. U.; YEH, L.; TERRY, N. Rate-limiting steps in selenium assimilation and volatilization by Indian Mustard.

Plant Physiology, Washington, v. 117, p. 1487-1494, 1998.

TURAKAINEN, M.; HARTIKAINEN, H.; SEPPANEN, M. Selenium in plants. In: EUROLA, M.;

HIETAMNIEMI, V. Twenty years of selenium

fertilization. Helsinkind: Agrifood, 2005. 108 p.

(Proceedings. Agrifood reports, 69). Disponível em:

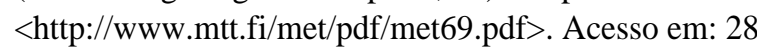
abr. 2007.
WHANGER, P. D. Selenocompounds in plants and animals and their biological significance. Journal of the American College of Nutrition, Corvalis, v. 21, n. 3,p. 223-232, 2002. Disponível em: <http:// Lwww.jacn.org/cgi/content/full1/21/3/223 3 . Acesso em: 15 jun. $200 \overline{7}$.

WHO, E. Selenpflanzen (Selenophyten). In: KINZEL, H. (Ed.). Pflanzenokologie und

Mineralstoffwechsel. Stuttgart: Verlag Eugen Ulmer, 1982. p. 511-519.

ZAYED, A.; LYTLE, C. M.; TERRY, N. Accumulation and volatilization of different chemical species of selenium by plants. Planta, Berlin, v. 206, p. 284-292, 1998. 